\title{
THETA FUNCTION IDENTITIES FROM OPTICAL NEURAL NETWORK TRANSFORMATIONS
}

\author{
E. ELIZALDE and A. ROMEO \\ Department E.C.M., Faculty of Physics \\ University of Barcelona \\ Diagonal 647 \\ 08028 Barcelona \\ Spain \\ (Received February 13, 1992 and in revised form May 12, 1992)

\begin{abstract}
We take a new approach to the generation of Jacobi theta function identities. It is complementary to the procedure which makes use of the evaluation of Parseval-like identities for elementary cylindrically-symmetric functions on computer holograms. Our method is more simple and explicit than this one, which was an outcome of the construction of neurocomputer architectures through the Heisenberg model.
\end{abstract}

KEY WORDS AND PHRASES. Jacobi theta functions, Riemann zeta functions, neural networks, computer holograms.

1991 AMS SUBJECT CLASSIFICATION CODES. 30B50, 78A60, 33A15.

Take the physical situation of optical holography described in Schempp [1]. A square-law detector encodes (in a massively parallel way) the optical path-length difference $x$ and the phase difference $y, x, y \in \mathbf{R}$, of two coherent signals of the same center frequency $\nu \neq 0$ and amplitudes $\varphi, \psi \in \mathcal{S}(\mathbf{R})$ (the Schwartz space of complex-valued $C^{\infty}$ amplitude functions on the real line, rapidly decreasing at infinity). Let $H(\varphi, \psi ; .,$.$) be the holographic transform of the writing$ amplitudes $\psi$ and $\varphi$

$$
H(\varphi, \psi ; ., .)=\int_{\mathbf{R}} d t e^{\imath 2 \pi y t} \psi(t-x) \bar{\varphi}(t)
$$

which describes, by coherent superposition, the holographic angle encoding. It is also convenient to define the auto-ambiguity function

$$
H(\psi ; ., .)=H(\psi, \psi ; ., .)
$$


According to the corollary of theorem 4 in Schempp [1], the elementary holograms $\left\{H\left(H_{m}, H_{n} ; ., .\right)\right\}_{m \geq 0, n \geq 0}$ form a Hilbert basis of the complex Hilbert space $L^{\mathbf{2}}(\mathbf{R} \oplus \mathbf{R})$, with

$$
H_{n}(t)=e^{-\frac{t^{2}}{2}} h_{n}(t)
$$

the Herrnite function of degree $n$ (i.e. $h_{n}(t)$ is the Hermite polynomial of degree $n$ ). The elementary holograms satisfy the Parseval-Plancherel type pixel identity

$$
\sum_{\left(\mu, \mu^{\prime}\right) \in \mathbf{Z}_{\oplus} \mathbf{Z}} H\left(\psi ; \mu, \mu^{\prime}\right) \bar{H}\left(\varphi ; \mu, \mu^{\prime}\right)=\sum_{\left(\mu, \mu^{\prime}\right) \in \mathbf{Z}_{\oplus \mathbf{Z}}}\left|H\left(\psi, \varphi ; \mu, \mu^{\prime}\right)\right|^{2} .
$$

The terms of this identity can be explicitly identified in two possible manners:

a) Taking advantage of the classical expression for the polynomials $h_{n}(t)$, like in Schempp [2]

b) Reasoning by theorem 7 in Schempp [1], which states that the elementary holograms admit the form

$$
H\left(H_{m}, H_{n} ; x, y\right)=\frac{(-1)^{n}}{\sqrt{m ! n !}} e^{\frac{-\pi\left(x^{2}+y^{2}\right)}{2}} \phi_{m, n}(\sqrt{\pi}|x+i y|),
$$

for all pairs $(x, y) \in \mathbf{R} \oplus \mathbf{R}$, where $\phi_{m, n}(X)$ denotes the matching polynomial in the variable $X$ associated to the bipartite graph $K_{m, n}$ :

$$
\phi_{m, n}(X)=\sum_{0 \leq l \leq\left\lfloor\frac{\frac{m+n}{2}}{2}\right]}(-1)^{l} c\left(K_{m, n}, l\right) X^{m+n-2 l},
$$

and the coefficients $c\left(K_{m, n}^{\prime}, l\right)$ are the number of disjoint synaptic interconnects of the 2-state unit neural network $K_{m, n}(m \geq n \geq 0)$ when activated by $l$ simultaneously firing neurons. This network has $m$ units in one state, $n$ in the other, and contains $l$ links between pairs in the two states, so that only one connection, at most, departs from or arrives to each unit.

Applying combinatorial analysis, we have found the general expression

$$
c\left(K_{m, n}, l\right)=C_{m, l} V_{n, l}=\frac{m !}{(m-l) ! ! !} \frac{n !}{(n-l) !} .
$$

In particular, for $m=n$ we obtain

$$
c\left(K_{m, m}, l\right)=\frac{(m !)^{2}}{[(m-l) !]^{2} l !} .
$$

Using these expressions, we recover the form of $\phi_{m, n}$ derived by the procedure a), which reads

$$
\phi_{m, n}(X)=(-1)^{n} m ! X^{m-n} e^{\frac{X^{2}}{2}} L_{n}^{(m-n)}\left(X^{2}\right),
$$

where

$$
L_{n}^{(m-n)}\left(X^{2}\right) \equiv e^{-\frac{X^{2}}{2}} L_{n}^{m-n}\left(X^{2}\right)
$$

being $L_{n}^{m-n}\left(X^{2}\right)$ the generalized Laguerre polynomial of degree $n$ and order $m-n$ in the variable $X^{2}$.

When applying (4) to these elementary holograms, we have to evaluate both sides of the relation

$$
\begin{gathered}
\sum_{\left(\mu, \mu^{\prime}\right) \in \mathbf{Z} \oplus \mathbf{Z}}(-1)^{m+n} e^{-\pi\left(\mu^{2}+\mu^{\prime 2}\right)} \phi_{m, m}\left(\sqrt{\pi}\left|\mu+i \mu^{\prime}\right|\right) \phi_{n, n}\left(\sqrt{\pi}\left|\mu+i \mu^{\prime}\right|\right) \\
=\sum_{\left(\mu, \mu^{\prime}\right) \in \mathbf{Z}_{\oplus} \mathbf{Z}} e^{-\pi\left(\mu^{2}+\mu^{\prime 2}\right)}\left|\phi_{m, n}\left(\sqrt{\pi}\left|\mu+i \mu^{\prime}\right|\right)\right|^{2} .
\end{gathered}
$$


These functions are actually connected with a type of theta functions, the so-called nil-theta functions, which may be defined in a natural way on a Heisenberg compact nilmanifold (see Auslander [3] and Mumford [4]). Different values of $m$ and $n$ for the preceding equality yield special identities whose final form may be written down as odd powers of $\pi$ in terms of theta-null values $\theta(0,1, k)=\sum_{n=-\infty}^{\infty} n^{k} e^{-\pi n^{2}}$. (We adopt the notation $\theta(a, \tau, k) \equiv \sum_{n=-\infty}^{\infty} n^{k} e^{-\pi \tau n^{2}+2 \pi n a}$ ). Thus, we obtain

$$
\begin{aligned}
& m=1, n=0 \quad \pi \theta(0,1,2) \quad=\frac{1}{4} \theta(0,1,0), \\
& m=2, n=1 \quad \pi^{3} \theta(0,1,6)=\frac{15}{32}\left[8 \pi^{2} \theta(0,1,4)-\theta(0,1,0)\right], \\
& m=3, n=2 \quad \pi^{5} \theta(0,1,10)=\frac{45}{64}\left[16 \pi^{4} \theta(0,1,8)-140 \pi^{2} \theta(0,1,4)+21 \theta(0,1,0)\right] \text {, } \\
& m=4, n=3 \quad \pi^{7} \theta(0,1,14)=\frac{91}{1024}\left[256 \pi^{6} \theta(0,1,12)-15840 \pi^{4} \theta(0,1,8)\right. \\
& \left.+166320 \pi^{2} \theta(0,1,4)-25245 \theta(0,1,0)\right] \text {, } \\
& m=5, n=4 \quad \pi^{9} \theta(0,1,18)=\frac{153}{1024}\left[256 \pi^{8} \theta(0,1,16)-58240 \pi^{6} \theta(0,1,12)\right. \\
& +4324320 \pi^{4} \theta(0,1,8)-45945900 \pi^{2} \theta(0,1,4) \\
& +6981975 \theta(0,1,0)] \text {, } \\
& m=6, n=5 \quad \pi^{11} \theta(0,1,22)=\frac{231}{8192}\left[2048 \pi^{10} \theta(0,1,20)-1240320 \pi^{8} \theta(0,1,16)\right. \\
& +338607360 \pi^{6} \theta(0,1,12)-25440901200 \pi^{4} \theta(0,1,8) \\
& \left.+270621351000 \pi^{2} \theta(0,1,4)-41128890075 \theta(0,1,0)\right] \text {, } \\
& m=7, n=6 \quad \pi^{13} \theta(0,1,26)=\frac{325}{16384}\left[4096 \pi^{12} \theta(0,1,24)-5440512 \pi^{10} \theta(0,1,20)\right. \\
& +3953892096 \pi^{8} \theta(0,1,16)-1092262691520 \pi^{6} \theta(0,1,12) \\
& +82160642163600 \pi^{4} \theta(0,1,8)-874071171873900 \pi^{2} \theta(0,1,4) \\
& +132842665857825 \theta(0,1,0)] \text {, }
\end{aligned}
$$

The first of these identities amounts to the following relation between holomorphic theta series:

$$
\frac{1}{4 \pi} \sum_{n=-\infty}^{\infty} e^{-\pi n^{2}}=\sum_{n=-\infty}^{\infty} n^{2} e^{-\pi n^{2}}
$$

which is a fundamental expression in the theory of Jacobi theta functions. As for the other identities, we have found a general and systematic way of producing them, which is much simpler and straightforward than the one stemming from network models. In fact, the last three expression were actually not explicitly obtained by Schempp: the necessary amount of time for it would had been prohibitive.

The idea behind our procedure is to take the general relationship (see, for example, Erdélyi [5])

$$
\theta(0, \tau, 0)=\frac{1}{\sqrt{\tau}} \theta\left(0, \frac{1}{\tau}, 0\right),
$$

of which the previous identity is the special case $\tau=1$. The function

$$
f(\tau) \equiv \theta(0, \tau, 0)-\frac{1}{\sqrt{\tau}} \theta\left(0, \frac{1}{\tau}, 0\right)=0
$$


is then identically equal to zero for every $\tau$. All their derivatives with respect to $\tau$ vanish as well. Thus, by considering $\frac{d}{d \tau} f(\tau)=0$ we get the fundamental expression

$$
-\pi \theta(0, \tau, 2)=-\frac{1}{2 \tau^{3 / 2}} \theta\left(0, \frac{1}{\tau}, 0\right)+\frac{\pi}{\tau^{5 / 2}} \theta\left(0, \frac{1}{\tau}, 2\right),
$$

which is another theta function identity for arbitrary $\tau$. In particular, by setting $\tau=1$ we recover the relation for $m=1, n=0$. However, taking the second derivative of $f(\tau)$ we obtain an identity that just repeats the previous one in (12). Only after evaluating $\left.\frac{d^{3}}{d \tau^{3}} f(\tau)\right|_{\tau=1}=0$ do we find the identity for $m=2, n=1$. Thus, the way of generating independent relations of this sort is to calculate

$$
\left.\frac{d^{k}}{d \tau^{k}} f(\tau)\right|_{\tau=1}=0, \text { for any odd integer } k .
$$

(Alternatively, we might restrict ourselves to the even derivatives as well, since the information contained in both sets of equations is the same).

Nevertheless, in order to obtain (12), the relations (17) have to be handled in a very specific manner. In fact, once we have derived one of these relationships, the highest order theta function occurring must be put in terms of the rest, and the result plugged into the next identity. This is the reason why in the expression for $\pi^{2 k+1}$ one only gets $\theta(0,1,2 k)$ on the l.h.s. and $\theta(0,1,2 k-$ 2), $\theta(0,1,2 k-6), \theta(0,1,2 k-10), \ldots, \theta(0,1,0)$ on the r.h.s.

Taking all this into account, we have written a REDUCE program that finds all the relevant identities, for $k$ up to an arbitrary value $k_{f}$. After every step, each new identity is written on the auxiliary file thetal.out. At the next iteration, the contents of this file is read by the source program, thus incorporating into memory the knowledge of the latest obtained relation. Actually, this program falls into the category of those that get fed from their own output. A further point to be made is that, for the algebraic handling of these expressions, REDUCE needs not know the explicit series form of $\theta(0, \tau, k)$. It is enough to supply it with the relations satisfied by the $\tau$-derivatives, that is

$$
\begin{aligned}
\frac{d}{d \tau} \theta(0, \tau, k) & =-\pi \theta(0, \tau, k+2), \\
\frac{d}{d \tau} \theta\left(0, \frac{1}{\tau}, k\right) & =\frac{\pi}{\tau^{2}} \theta(0, \tau, k+2) .
\end{aligned}
$$

A listing of the source file is given in the PCREDUCE version (Table 1). In the example chosen we have taken $k_{f}=7$, although this value may be arbitrarily changed.

A general formula can be derived for the first and for the last coefficients of the r.h.s of the theta identities (12). Calling these coefficients $a_{k}$ and $b_{k}$, respectively, we have found

$$
a_{k}=\frac{k(2 k-1)}{4}, \quad b_{k}=2^{-k-1}\left[2\left(\frac{k-1}{2}\right)^{2}-1\right] \prod_{j=1}^{k-1}(2 j+1) .
$$

The rest of the coefficients are given by more tricky combinations of this same kind - where some of the factors of the multi-product (either the ones from the begining or from the end) are missing. From a practical point of view, it is more straightforward to rely directly on the recurrent derivation and on the REDUCE program which yields explicitly any of the identites (up to any finite, reasonable order) in a resonable amount of time.

Further research along this line will be aimed at the derivation of new identities involving $\theta(a, 1, k), a \neq 0$, and also multidimensional theta functions. 


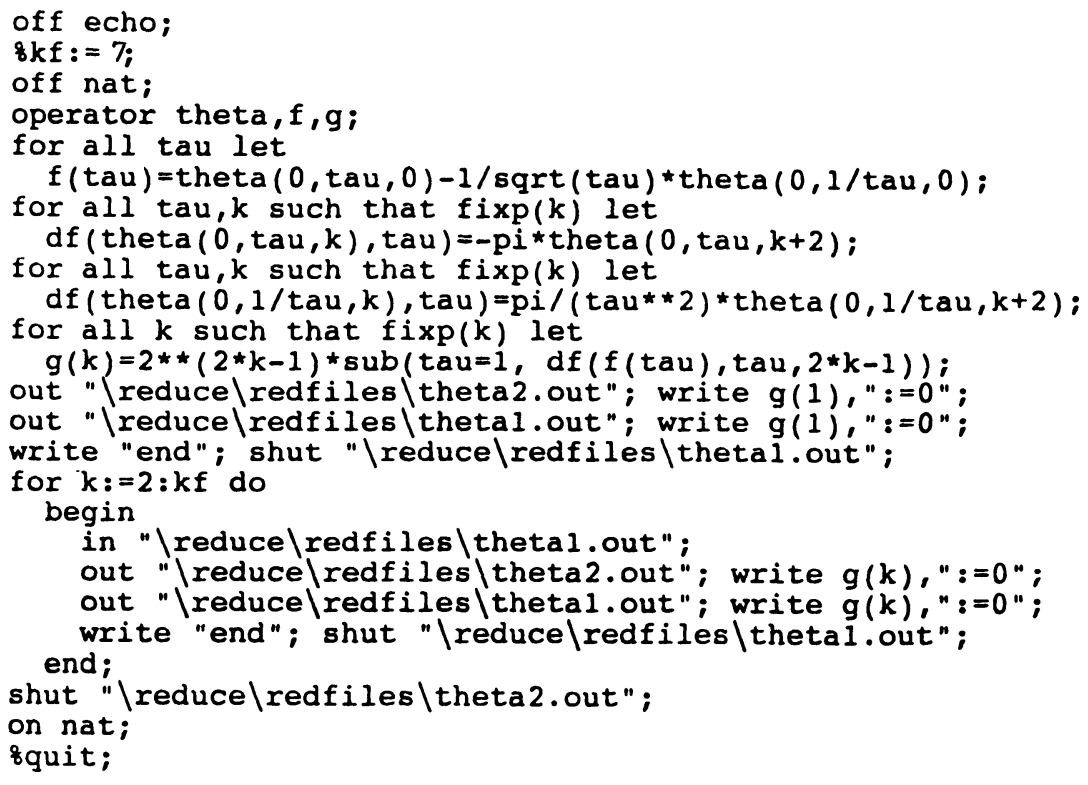

Table 1. A listing of the PCREDUCE source file.

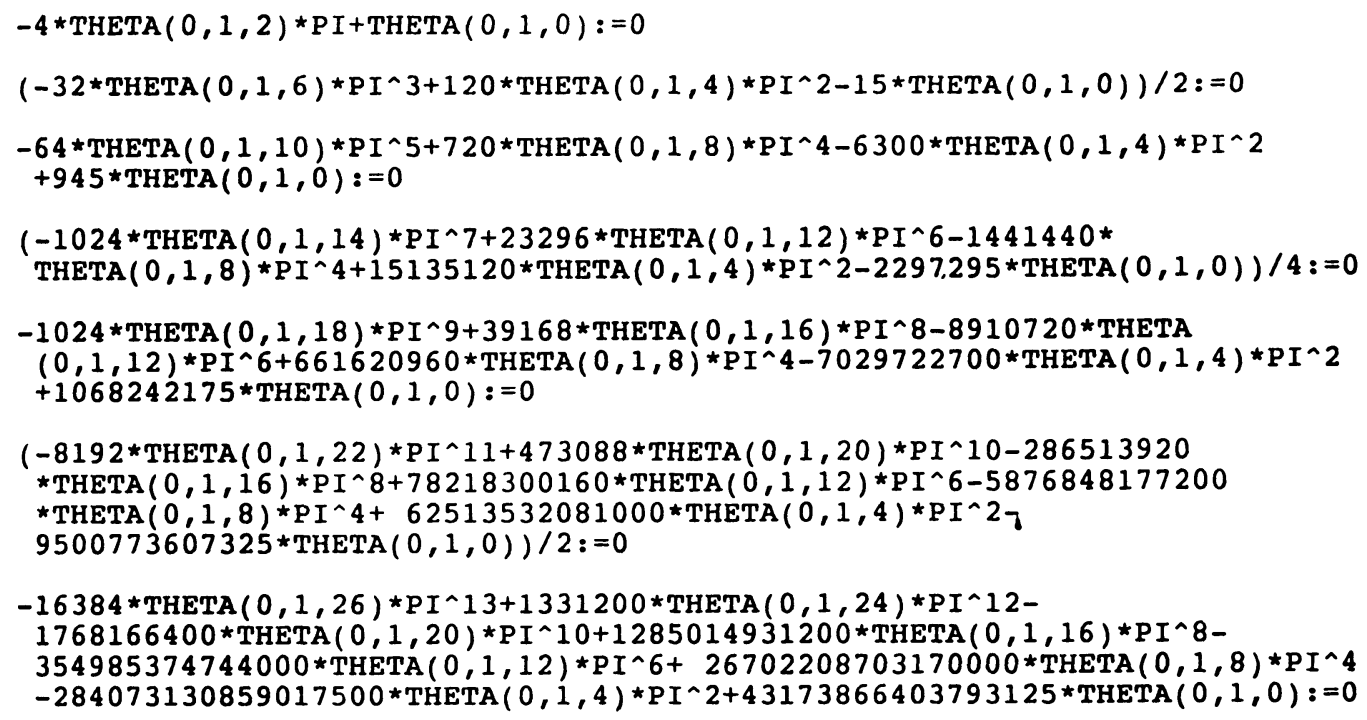

Table 2. The successive theta function identities. 
Acknowledgments. We thank Prof. W. Schempp for sending us his papers, which inspired the present investigation. This work has been supported by Dirección General de Investigacion Científica y Técnica (DGICYT), research project PB90-0022. AR has also obtained financial help through research fellowship from the Spanish Ministry of Education and Science. Logistic help under the form of a Donation of the Alexander von Humboldt-Foundation is also gratefully acknowledged.

\section{References}

[1] W. Schempp, Neurocomputer architectures, Results in Math. 16, 103-110, 1989.

[2] W. Schempp, Radar ambiguity functions, the Heisenberg group, and holomorphic theta series, Proc. Amer. Math. Soc. 92, 345-382, 1984.

[3] L. Auslander, Lectures on nil-theta functions, CBMS Regional Conf. Ser. in Math. 34, Amer. Math. Soc., Providence, R.I., 1977.

[4] D. Mumford, Thata lectures on theta I, Progress in Math., vol. 28, Birkhäuser, Boston, Mass., 1983.

[5] A. Erdélyi, Ed., Higher transcendental functions, vol. I, McGraw-Hill, New York, 1953. 


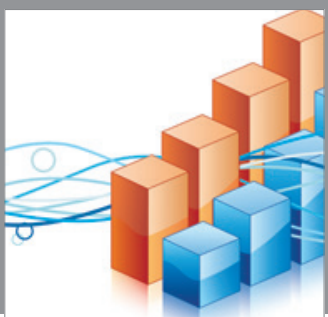

Advances in

Operations Research

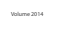

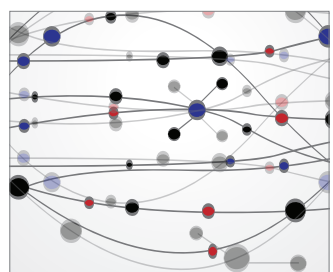

\section{The Scientific} World Journal
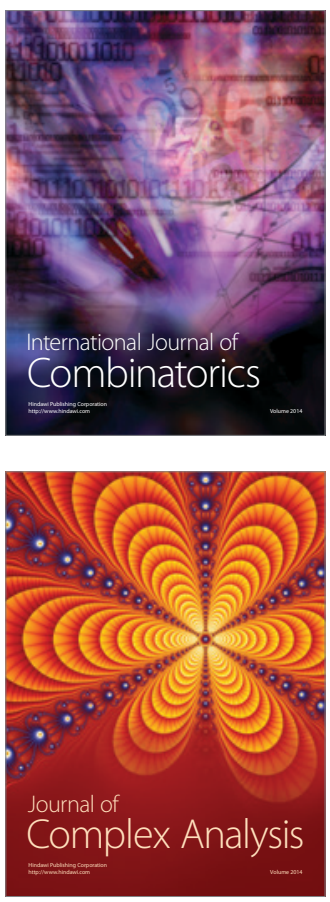

International Journal of

Mathematics and

Mathematical

Sciences
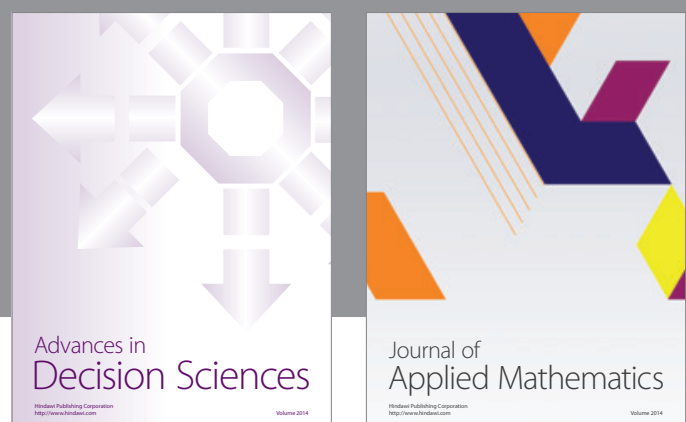

Journal of

Applied Mathematics
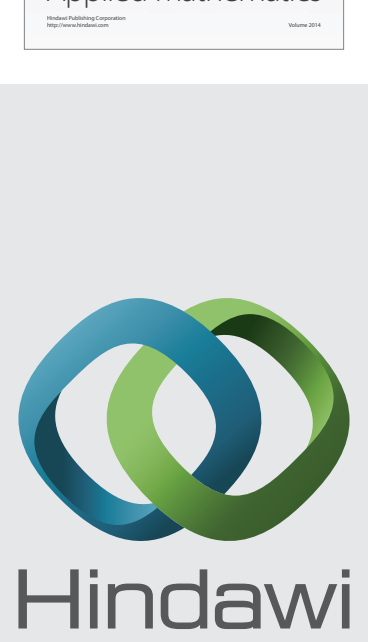

Submit your manuscripts at http://www.hindawi.com
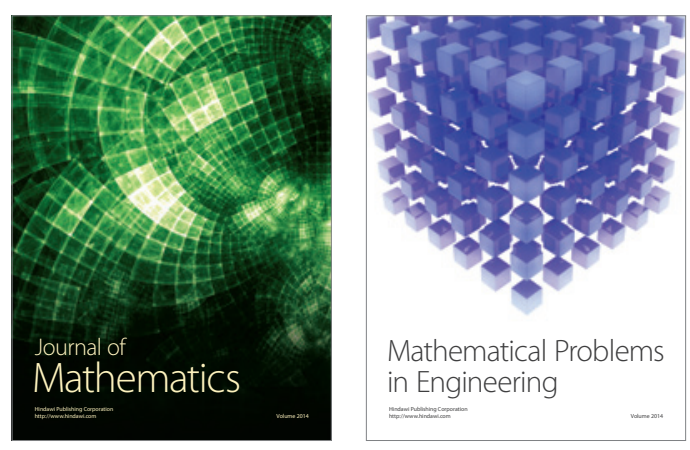

Mathematical Problems in Engineering
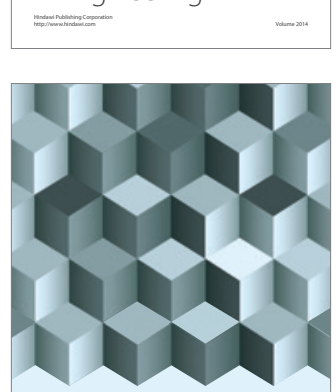

Journal of

Function Spaces
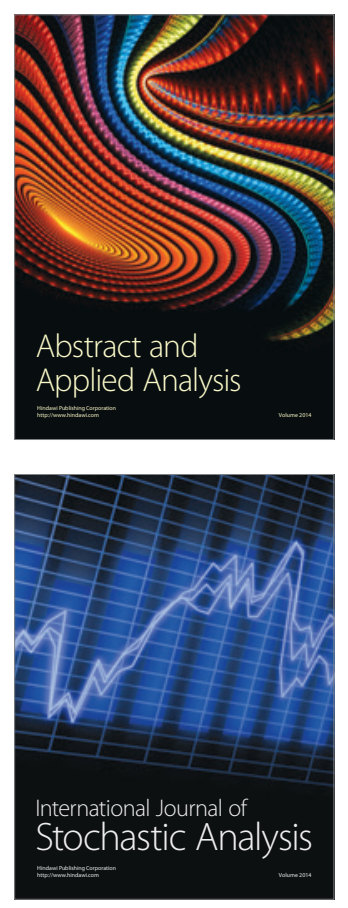

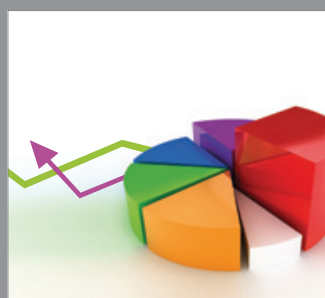

ournal of

Probability and Statistics

Promensencen
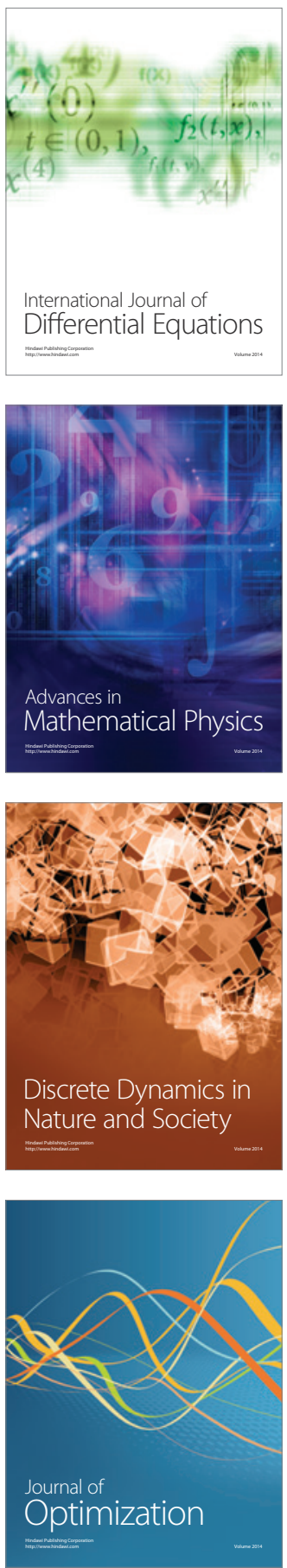